\title{
DECAY AND BOUNDEDNESS RESULTS FOR A MODEL OF LAMINAR FLAMES WITH COMPLEX CHEMISTRY
}

\author{
JOEL D. AVRIN
}

(Communicated by Barbara L. Keyfitz)

\begin{abstract}
We consider the reaction-diffusion equations modeling two-step reactions with Arrhenius kinetics on bounded spatial domains or over all of $\mathbb{R}^{n}$. After noting the existence, uniqueness, and nonnegativity of global strong solutions with virtually arbitrary nonnegative initial data, we give conditions on the initial temperature that guarantee decay of the concentrations to zero and a supremum norm bound on the temperature. In our first such result we assume that the initial temperature $T_{0}$ is uniformly bounded above the two ignition temperatures. Specializing to the case of bounded spatial domains, we replace this condition by the more general requirement that the average of $T_{0}$ over the domain is above both ignition temperatures. Finally, we note a boundedness result with equal diffusion coefficients that holds for arbitrary choices of the other parameters. Combining this assumption with the hypotheses, noted above, about the initial temperature, we obtain steady-state convergence results for the temperature as well as the concentrations.
\end{abstract}

\section{INTRODUCTION}

The following system of reaction-diffusion equations arises as a model of laminar flames with complex chemistry corresponding to the two-step reaction $A \rightarrow B \rightarrow C$ :

$$
\begin{gathered}
T_{t}=d_{0} \Delta T+Q_{1} Y_{1} f_{1}(T)+Q_{2} Y_{2} f_{2}(T) \\
Y_{1 t}=d_{1} \Delta Y_{1}-Y_{1} f_{1}(T) \\
Y_{2 t}=d_{2} \Delta Y_{2}+Y_{1} f_{1}(T)-Y_{2} f_{2}(T) .
\end{gathered}
$$

Here $T$ is the dimensionless temperature, $Y_{1}$ is the concentration of $A$, and $Y_{2}$ the concentration of $B . T, Y_{1}$, and $Y_{2}$ depend on $x$ and $t$ where $(x, t) \in$ $\Omega \times \mathbb{R}^{+}$with $\Omega=\mathbb{R}^{n}$ or a bounded domain in $\mathbb{R}^{n}$ with smooth boundary. Both $d_{i}$ and $Q_{j}$ are positive constants, and the functions $f_{j}$ take the form of Arrhenius rate laws; there exist positive constants $B_{j}$ and $E_{j}$ and nonnegative

Received by the editors July 25, 1989 and, in revised form, December 11, 1989.

1980 Mathematics Subject Classification (1985 Revision). Primary 35B40, 35K57, 80A25. 
constants $T_{j}$ such that for $j=1,2$

$$
f_{j}(T)=\left\{\begin{array}{l}
0, T<T_{j} \\
B_{j} \exp \left(-E_{j} /\left(T-T_{j}\right)\right), T \geq T_{j} .
\end{array}\right.
$$

In (1.2), $T_{j}$ represents ignition temperature. For the physical background behind equations (1.1) and (1.2), see, e.g., [4, 12].

The one-step reaction $A \rightarrow B$ is modeled by (1.1a) and (1.1b) with $Y_{2}=0$. The existence of traveling-wave solutions with $\Omega=\mathbb{R}$ was established in [3] for $T_{1}>0$ and in [9] for $T_{1}=0$. These traveling-wave solutions have been shown to be stable if the Lewis number $L=d_{1} / d_{0}$ is close to 1 , while they are unstable if $L$ is far from 1 and the activation energy $E_{1}$ is large. These results have been shown by formal asymptotics in [5] and [7]; a rigorous proof of the instability result has recently been obtained ([11]). Qualitative behavior for the general Cauchy problem for the one-step reaction was developed in [1] for $\Omega=\mathbb{R}$ and in [2] for the case in which $\Omega$ is a bounded domain in $\mathbb{R}^{n}$ with various boundary conditions prescribed. In particular, examples of flame propagation versus flame quenching are discussed in [1] and [2].

About the full two-step reaction less is known. Two flame fronts may propagate, each corresponding to a different stage in the reaction, and each proceeding with a different velocity. The existence of traveling-wave solutions was established in [10] in the case that the second front is faster than the first. Stability results do not as yet exist, but one expects, from the one-step example, that instability of traveling waves occurs for a wide choice of parameters.

As in the one-step case, it is thus of interest to study the general Cauchy problem for (1.1) when $\Omega=\mathbb{R}$, and it is of independent interest to study the Cauchy/boundary-value problem for (1.1) on a bounded domain $\Omega$ in $\mathbb{R}^{n}$ with smooth boundary. We consider both of these Cauchy problems in this paper for arbitrary nonnegative, bounded, and uniformly continuous initial data $T_{0}(x)=T(x, 0), Y_{10}(x)=Y_{1}(x, 0), Y_{20}(x)=Y_{2}(x, 0)$, where in the boundeddomain case we assume zero Neumann boundary conditions for $T, Y_{1}$, and $Y_{2}$. As we will see in the last part of this section, it is straightforward to establish the existence, uniqueness, and regularity of global strong solutions to (1.1) for each choice of initial data described above (Theorem 1.1).

In our first main result (Theorem 2.1) we show that if there exists a constant $\alpha$ such that $T_{0}(x) \geq \alpha>T_{j}$ for all $x$ in $\Omega$ and $j=1,2$, then $T$ remains bounded and both $Y_{1}$ and $Y_{2}$ decay exponentially to zero.

In $\S 3$ we restrict to the bounded-domain case while relaxing the condition on $T_{0}$. Requiring only that the average of $T_{0}$ over $\Omega$ [see (3.1)] be greater than or equal to $\alpha$ (with $\alpha>T_{j}$ ), we show that eventually $Y_{1}$ and $Y_{2}$ decay to zero at exponential rates and that $T$ remains bounded. The result in $\S 2$ will be a key component of that proof.

Both of these theorems can be recast in an abstract setting that allows for more general $f_{j}$ than those specified by (1.2). It will be clear from the proofs that it suffices for the $f_{j}$ to be nonnegative, bounded, smooth, and monotone 
increasing and for there to exist nonnegative constants $T_{j}$ such that $f(T)>0$ for $T>T_{j}$; see, e.g., (2.1) and (2.2) below.

Finally, in $\S 4$ we show that if $d_{0}=d_{1}=d_{2}$, then $T, Y_{1}$, and $Y_{2}$ remain bounded regardless of the values of the other parameters. Suppose in addition we assume the conditions on $T_{0}$ imposed in Theorem 2.1 in the case $\Omega=\mathbb{R}$, or the conditions on $T_{0}$ imposed in Theorem 3.1 in the bounded-domain case; for $\Omega=\mathbb{R}$ we also assume that $T_{0}, Y_{10}$, and $Y_{20}$ have limits at $\pm \infty$. We already have under these conditions that $Y_{1}$ and $Y_{2}$ converge uniformly to the zero steady state; we show in addition that $T$ converges to a constant steady state specified by $T_{0}, Y_{10}$, and $Y_{20}$. The convergence for $T$ is uniform in the bounded domain case and uniform on compact sets in the case $\Omega=\mathbb{R}$.

We close this section with a discussion of the aforementioned existence, uniqueness, nonnegativity, and regularity of general solutions to (1.1). Global existence, uniqueness, and regularity in $t$ and $x$ for $t$ positive follow immediately from the fact that the nonlinear terms in (1.1) are smooth and in particular globally Lipschitz continuous as functions of $T$ and $Y$. Nonnegativity of solutions follows by applying Theorem 14.3 of [8]. One can also deduce nonnegativity directly from (1.1) by first observing that the evolution of $Y_{1}$ is governed by a positivity-preserving fundamental solution. One can then write an integral equation for $Y_{2}$ in terms of a similar positivity-preserving fundamental solution and the (nonnegative) forcing function $Y_{1} f_{1}(T)$. Nonnegativity for $T$ then follows directly from its standard integral equation in terms of $\exp \left(t d_{0} \Delta\right)$. In any case we thus have the following result; here $C_{B U}(\Omega)$ indicates the uniformly continuous and bounded functions on $\Omega$ :

Theorem 1.1. Let $\Omega=\mathbb{R}^{n}$ or a bounded domain in $\mathbb{R}^{n}$ with smooth boundary. In the latter case, let $\Delta$ be equipped with zero Neumann boundary conditions. Then for arbitrary nonnegative initial data $T_{0}, Y_{10}, Y_{20} \in C_{B U}(\Omega)$ there exist unique global strong solutions $T, Y_{1}$, and $Y_{2}$ of (1.1) such that $T, Y_{1}, Y_{2} \in$ $C\left([0,+\infty) ; C_{B U}(\Omega)\right) \cap C^{j}\left((0,+\infty) C^{k}(\Omega)\right)$ for any $j, k \geq 1$.

\section{A DECAY AND BOUNDEDNESS RESULT}

Theorem 2.1. Let $T, Y_{1}$, and $Y_{2}$ be as in Theorem 1.1. Assume in addition that there exists a constant $\alpha$ such that $T_{0}(x) \geq \alpha>T_{j}$ for all $x$ in $\Omega$ and $j=1,2$. Then $Y_{1}$ and $Y_{2}$ decay exponentially to zero and $T$ remains bounded. Proof. We have from (1.1a), standard comparison principles (see, e.g., [6]), and the nonnegativity of $Q_{j}, Y_{j}$, and $f_{j}(T)$ that for all $x$ in $\Omega$ and $t \geq 0$

$$
T(x, t) \geq\left(\exp \left(t d_{0} \Delta\right) T_{0}\right)(x) \geq \alpha .
$$

Set $\beta_{1}=f_{1}(\alpha)$ and $\beta_{2}=f_{2}(\alpha)$. Then, for all $x$ in $\Omega$ and $t \geq 0$,

$$
f_{j}\left(T_{j}(x, t)\right) \geq \beta_{j}, j=1,2
$$

by the monotonicity of $f_{j}$ (see (1.2)). Standard semigroup theory (or a com- 
parison principle) then implies that

$$
\left\|Y_{1}(t)\right\|_{\infty} \leq\left\|Y_{10}\right\|_{\infty} e^{-\beta_{1} t}
$$

for all $t \geq 0$.

Now let $U_{2}(t, s)$ be the fundamental solution generated by the operator $d_{2} \Delta-V_{2}(t)$, where $V_{2}(t)$ denotes multiplication by $f_{2}(T(t))$. We have that

$$
Y_{2}(t)=U_{2}(t, 0) Y_{20}+\int_{0}^{t} U_{2}(t, s) Y_{1}(s) f_{1}(T(s)) d s,
$$

and again from (2.2) that

$$
\left\|U_{2}(t, s)\right\|_{\infty} \leq e^{-\beta_{2}(t-s)},
$$

where the left-hand side of $(2.5)$ denotes the operator norm of $U_{2}(t, s)$ taken over $C_{B U}(\Omega)$. Combining (2.3), (2.4), and (2.5) with (1.2), we then have that

$$
\begin{aligned}
\left\|Y_{2}(t)\right\|_{\infty} & \leq\left\|Y_{20}\right\|_{\infty} e^{-\beta_{2} t}+\int_{0}^{t} e^{-\beta_{2}(t-s)}\left\|Y_{1}(s) f_{1}(T(s))\right\|_{\infty} d s \\
& \leq\left\|Y_{20}\right\|_{\infty} e^{-\beta_{2} t}+\left\|Y_{10}\right\|_{\infty} B_{1} \int_{0}^{t} e^{-\beta_{2}(t-s)} e^{-\beta_{1} s} d s .
\end{aligned}
$$

Select $\gamma>0$ such that $\beta_{2} \geq \gamma$ and $\beta_{1}>\gamma$, then

$$
e^{-\beta_{2}(t-s)}=e^{-\left(\beta_{2}-\gamma\right)(t-s)} e^{-\gamma(t-s)} \leq e^{-\gamma(t-s)}
$$

so from (2.6) we have that

$$
\begin{aligned}
\left\|Y_{2}(t)\right\|_{\infty} & \leq\left\|Y_{20}\right\|_{\infty} e^{-\beta_{2} t}+\left\|Y_{10}\right\|_{\infty} B_{1} e^{-\gamma t} \int_{0}^{t} e^{-\left(\beta_{1}-\gamma\right) s} d s \\
& \leq K_{2} e^{-\gamma t}
\end{aligned}
$$

where

$$
K_{2}=\left\|Y_{20}\right\|_{\infty}+\left\|Y_{10}\right\|_{\infty} B_{1}\left(1 /\left(\beta_{1}-\gamma\right)\right.
$$

Thus $Y_{1}(t)$ and $Y_{2}(t)$ decay exponentially to zero in $t$. Setting $W_{0}(t)=$ $\exp \left(t_{0} \Delta\right)$ we know that

(2.10) $T(t)=W_{0}(t) T_{0}+\int_{0}^{t} W_{0}(t-s)\left[Q_{1} Y_{1}(s) f_{1}(T(s))+Q_{2} Y_{2}(s) f_{2}(T(s))\right] d s$.

Applying supremum norms to both sides of (2.10) and using (1.2), (2.3), (2.8), and (2.9), we then have that

$$
\|T(t)\|_{\infty} \leq\left\|T_{0}\right\|_{\infty}+Q_{1}\left\|Y_{10}\right\|_{\infty} B_{1}\left(1 / \beta_{1}\right)+Q_{2} K_{2} B_{2}(1 / \gamma)
$$

for all $t \geq 0$, thus completing the proof of the theorem.

3. FURTHER DECAY AND BOUNDEDNESS RESULTS ON BOUNDED DOMAINS

We define the average $T_{A V}$ of $T_{0}$ over $\Omega$ as follows:

$$
T_{A V}=1 /(|\Omega|) \int_{\Omega} T_{0}(x) d x
$$


where $|\Omega|$ is the volume of $\Omega$. We are assuming that $\Delta$ is equipped with zero Neumann boundary conditions; i.e., the domain of $\Delta$ is the closure in $C(\bar{\Omega})$ of the $C^{2}$ functions $u$ on $\Omega$ such that

$$
\frac{\partial u}{\partial \nu}=0 \text { on } \partial \Omega
$$

where $\nu$ is the outward normal on the boundary $\partial \Omega$. Let $W_{0}(t)=\exp \left(t d_{0} \Delta\right)$. Then it follows that

$$
\lim _{t \rightarrow \infty}\left(W_{0}(t) T_{0}\right)(x)=T_{A V}
$$

uniformly on $\Omega$ for $T_{0}$ as in Theorem 1.1. That (3.3) holds for $C^{1}$ functions $T_{0}$ can be seen by expanding $W_{0}(t) T_{0}$ in terms of the eigenfunctions of $\Delta$ : the first eigenfunction is the constant $1 /|\Omega|$, with eigenvalue 0 ; thus, the first term in the expansion is $T_{A V}$. The rest of the superposition is bounded by a constant times $\exp \left(-\lambda_{2} t\right)$ where $\lambda_{2}$ is the second (positive) eigenvalue of $-\Delta$. The result for general $T_{0}$ follows, using the regularity of $T(t)$ for $t>0$ and the mass conservation property of the heat equation holding for $\Delta$ as above.

The next result then follows easily from these observations and Theorem 2.1:

Theorem 3.1. Under the conditions of Theorem 1.1 in the case that $\Omega$ is bounded, assume in addition that $T_{A V}>T_{j}, j=1,2$. Then $T$ remains bounded and there exist positive constants $t_{0}, \beta_{1}, \beta_{2}$, and $K_{2}$ such that for all $t \geq t_{0}$

$$
\begin{aligned}
& \left\|Y_{1}(t)\right\|_{\infty} \leq\left\|Y_{10}\right\|_{\infty} e^{-\beta_{1}\left(t-t_{0}\right)}, \\
& \left\|Y_{2}(t)\right\|_{\infty} \leq K_{2} e^{-\beta_{2}\left(t-t_{0}\right)} .
\end{aligned}
$$

Proof. From the remarks concerning (3.3) we have that, given $\alpha$ with $T_{A V}>$ $\alpha>T_{j}, j=1,2$, that there exists a $t_{0}>0$ such that $t \geq t_{0}$ implies for all $x$ in $\Omega$ that $T(x, t) \geq \alpha$. We then obtain (3.4) by regarding $T\left(t_{0}\right), Y_{1}\left(t_{0}\right), Y_{2}\left(t_{0}\right)$ as initial data for (1.1) and noting that $\left\|Y_{1}\left(t_{1}\right)\right\|_{\infty} \leq\left\|Y_{10}\right\|_{\infty}$ by the maximum principle. The boundedness of $T$ then basically follows as in Theorem 2.1.

\section{BOUNDEDNESS AND STEADY-STATE CONVERGENCE WITH EQUAL DIFFUSION COEFFICIENTS}

We assume in this section that $d_{i}=1$ for $i=0,1,2$. The next result follows easily, using only a slight modification of a simple argument used in [10] in the case of equal diffusion coefficients; see also $\S 7$ of [1] and $\S 3$ of [2] for further applications in the one-step case of this argument.

Theorem 4.1. Set $d_{i}=1$ for $i=0,1,2$. Then $T, Y_{1}$, and $Y_{2}$ remain bounded for arbitrary choices of the other parameters.

Proof. Let $W=T+\left(Q_{1}+Q_{2}\right) Y_{1}+Q_{2} Y_{2}$. Then, by adding (1.1a)-(1.1c), we obtain that $W_{t}=\Delta W$. By nonnegativity of $T, Y_{1}, Y_{2}$ we then have that

$$
0 \leq T+\left(Q_{1}+Q_{2}\right) Y_{1}+Q_{2} Y_{2} \leq T_{0}+\left(Q_{1}+Q_{2}\right) Y_{10}+Q_{2} Y_{20},
$$

and the theorem is established. 
Corollary 4.1. Suppose $\Omega=\mathbb{R}$ and that $T_{0}, Y_{10}$, and $Y_{20}$ have limits at $\pm \infty$. Then under the conditions of Theorem 2.1 and Theorem 4.1 we have that $Y_{1}(t)$ and $Y_{2}(t)$ converge uniformly to zero and that $T(t)$ converges on compact spatial sets to the average of the values of $T_{0}+\left(Q_{1}+Q_{2}\right) Y_{10}+Q_{2} Y_{20}$ at $+\infty$ and $-\infty$. Proof. Let $W$ be as in the proof of Theorem 4.1. Then $W(t)$ converges on compact spatial sets to the constant indicated by a well-known property of $e^{t \Delta}$ (see, e.g., Lemma 5.2 of [1]). The result for $T(t)$ then follows by the exponential decay to zero of $Y_{1}(t)$ and $Y_{2}(t)$.

Corollary 4.2. Suppose $\Omega$ is a bounded domain in $\mathbb{R}^{n}$ with smooth boundary. Then, under the conditions of Theorem 3.1 and Theorem 4.1, $Y_{1}(t)$ and $Y_{2}(t)$ converge uniformly to zero and $T(t)$ converges uniformly to the average of $T_{0}+$ $\left(Q_{1}+Q_{2}\right) Y_{10}+Q_{2} Y_{20}$ over $\Omega$.

Proof. We use the same proof as in Corollary 4.1 except that $W(t)$ converges to the indicated constant by the remarks preceding the proof of Theorem 3.1.

\section{REMARKS}

Note that the condition $T_{A V}>T_{j}, j=1,2$ of $\S 3$ is quite general and supercedes the conditions imposed in $\S 2$ when $\Omega$ is bounded. It is a reasonable condition to impose, since in practice the $T_{j}$ are small numbers while the burn temperature is typically very large.

The main applications of Theorem 2.1 are thus to serve as a key ingredient in the proof of Theorem 3.1 and to handle the case $\Omega=\mathbb{R}^{n}$ (e.g., $n=1$ ). The assumption $T_{0}(x) \geq \alpha>T_{j}$ for all $x$ in $\mathbb{R}$ is quite restrictive; it requires, as for example in the case of a premixed reactive gas, that the gas be already hot when it enters the chamber. This occurs, for example, in an engine equipped with a precombustion chamber, so the condition on $T_{0}$ imposed in Theorem 2.1 is not without physical application.

One can imagine trying to generalize Theorem 2.1 for $\Omega=\mathbb{R}$ along the lines of Theorem 6.1 in [1], where it is shown in the one-step case that suitable decay of the concentration to zero, roughly mimicking flame-front propagation, is established whenever the average of $T_{0}$ at $+\infty$ and $-\infty$ is above ignition temperature. Note the analogy with the condition on $T_{A V}$ imposed here in $\S 3$; again it is a reasonable condition to impose, and in particular allows ignition to occur at one end only for a wide class of initial data.

Extending this result to the two-step case is complicated by the presence of the nonnegative term $Y_{1} f_{1}(T)$ in (1.1c). Detailed estimates showing that this term decays fast enough to be integrable in $t$ may be needed, along with an addressing of the relationships among $T_{1}, T_{2}, Q_{1}$, and $Q_{2}$. Such considerations are beyond the scope of the analysis in [1] and the present work.

The main ingredients of the proof of Theorem 3.1 appeared first in application to the one-step case on bounded domains in $\S 3$ of [2]. Note once again that Theorem 2.1 allows the application of Theorem 3.1 to the two-step case. 


\section{REFERENCES}

1. J. D. Avrin, Qualitative theory for a model of laminar flames with arbitrary nonnegative initial data, J. Differential Equations 84 (1990), 290-308.

2. __ Qualitative theory of the Cauchy problem for a one-step reaction model on bounded domains, submitted.

3. H. Berestycki, B. Nicolaenko, and B. Scheurer, Traveling wave solutions to combustion models and their singular limits, SIAM J. Math. Anal. 16 (1985), 1207-1242.

4. J. Buckmaster and G. S. S. Ludford, Theory of laminar flames, Cambridge University Press, Cambridge, 1982.

5. P. Clavin, Dynamical behavior of premixed fronts in laminar and turbulent flows, Progr. Energy Comb. Sci. 11 (1985), 1-59.

6. M. H. Protter and H. F. Weinberger, Maximum principles in differential equations, PrenticeHall, Englewood Cliffs, NJ, 1967.

7. G. I. Sivashinsky, Instabilities, pattern formation, and turbulence in flames, Ann. Rev. Fluid Mech. 15 (1988), 179-199.

8. J. Smoller, Shock waves and reaction-diffusion equations, Springer-Verlag, Berlin, Heidelberg, and New York, 1983.

9. D. Terman, Connection problems arising from nonlinear diffusion equations, Proc. Microconference on Nonlinear Diffusion (J. Serrin, L. Peletier, W.-M. Ni, eds.), Berkeley, California, 1986.

10. __ An application of the Cauchy index to combustion, Dynamics of Infinite Dimensional Systems, NATO AS1 series, vol. F37 (S.-N. Chow, J. K. Hale, eds.) Springer-Verlag, Berlin and Heidelberg, 1987.

11. Stability of planar wave solutions to a combustion model, preprint.

12. F. Williams, Combustion theory, 2nd ed., Addison-Wesley, Reading, MA, 1985.

Department of Mathematics, University of North Carolina at Charlotte, CharLOTTE, NORTH CAROLINA 28223 